\section{Zwölf-Marker-Algorithmus berechnet Aktivität der rheumatoiden Arthritis}

Um die rheumatoide Arthritis (RA) optimal zu behandeln, ist es erforderlich, die Krankheitsaktivität möglichst genau abzuschätzen. Ein Algorithmus, in den die Werte von zwölf Biomarkern aus dem Serum einfließen, kann dabei helfen, wie die Ergebnisse einer holländisch-amerikanischen Studie nahelegen.

Z u den am besten validierten Messinstrumenten der Krankheitsaktvität der RA zählen der Disease Activity Score 28 (DAS28) und seine Varianten, die zusätzlich die Blutsenkung (DAS28ESR) oder das C-reaktive Protein (DAS28-CRP) verwenden. So nützlich sie sind, haben sie doch Schwächen: Die klinische Beurteilung ist subjektiv, und die Entzündungsmarker ESR und CRP sind nicht RA-spezifisch.

Weil kein einzelner Biomarker die komplexe Biologie der RA abbildet, wurde ein Algorithmus entwickelt, der zwölf solcher Marker umfasst: Serum-Amyloid A, Interleukin 6, Tumor-NekroseFaktor-Rezeptor Typ I, vaskulärer Endo- thelwachstumsfaktor A, Matrix-Metallopeptidase 1 und 3, Knorpel-Glykoprotein 39, epidermaler Wachstumsfaktor, vaskuläres Zelladhäsionsmolekül 1, Leptin, Resistin und C-reaktives Protein. Der Algorithmus produziert daraus einen Summenwert (Multiple Biomarker Disease Activity [MBDA] Score) zwischen 1 und 100 . Werte bis 25 stehen für eine Remission, solche von 26-29 für eine niedrig aktive RA. Bei einem Score von 30-44 ist die RA mäßig aktiv, bei Werten über 44 hoch aktiv. In der Studie wurde die Aussagekraft des MBDAScore untersucht. Analysiert wurden dafür Daten der CAMERA-Studie. Als Referenz diente der DAS28-CRP.
Einen DAS28-CRP-Wert einer niedrig aktiven Erkrankung zugrunde gelegt, ergaben sich signifikante Korrelationen zwischen MBDA- und DAS28-CRP-Ergebnissen; je nach statistischem Berechnungsverfahren lagen die Koeffizienten zwischen 0,4 und 0,86 ( $p<0,001)$. Damit bestand eine akzeptable bis hohe Korrelation. Allerdings gelang es mit dem MBDA-Algorithmus ebenso wenig wie mit dem DAS28-CRP, eine radiologische Progression in statistisch zuverlässiger Weise vorherzusagen $(p=0,09)$.

Fazit: Der MBDA-Algorithmus produziert zuverlässig ähnliche Resultate wie der etablierte DAS28-CRP. Bevor er Eingang in den Versorgungsalltag findet, bedarf es allerdings einer weiteren Validierung. Beispielsweise ist zu klären, wie sich die Vorhersage einer radiologischen Progression verbessern ließe.

Dr. Robert Bublak

Bakker MF et al. Performance of a multi-biomarker score measuring rheumatoid arthritis disease activity in the CAMERA tight control study. Ann Rheum Dis 2012; 71: 1692-7

\title{
Reizdarm schwächt auch die Knochen
}

\section{Chronisch entzündliche Darmerkrankungen (CED) erhöhen bekanntlich das Risiko, eine Osteoporose zu entwickeln. Ob und in welchem Maß das auch für das Reizdarmsyndrom (RDS) gilt, haben US-amerikanische Forscher untersucht.}

D en Ergebnissen einer Studie unter der Leitung von Derrick Stobaugh, NorthShore University Health System in Evanston (Illinois)zufolge tragen RDSPatienten im Vergleich zu Personen ohne RDS ein mehr als vierfach höheres Osteoporoserisiko (Odds Ratio [OR] 4,28). Dadurch bedingte Frakturen kommen bei ihnen mehr als doppelt so häufig vor (OR 2,36). Besonders oft brechen sie sich die Handgelenke (OR 2,41).

Stobaugh und sein Team hatten für ihre Untersuchung auf die Datensammlung des Nationwide Emergency Department Sample (NEDS) zurückgegriffen. In ihr sind mehr als 28 Millionen Fälle von Patienten verzeichnet, die in einer Notaufnahme versorgt werden mussten. Knapp 320.000 wiesen eine RDS-Diagnose auf, rund 18.000 von die- sen litten zusätzlich an Osteoporose. Pathologische Frakturen des Handgelenks, der Hüfte oder eines Wirbels waren bei etwa 700, traumatische Brüche bei 1.500 Patienten festgestellt worden.

Das Osteoporoserisiko, das mit einer RDS-Diagnose einherging, übertraf jenes von Morbus-Crohn- und Colitis-ulcerosa-Patienten (OR 2,85 bzw. 3,62). Eine Zöliakie war indessen noch häufiger mit verminderter Knochendichte assoziiert (OR 6,41). Ähnlich verhielt es sich bei der durch Osteoporose bedingten Frakturgefahr. Auch hier waren Patienten mit Morbus Crohn und Colitis ulcerosa weniger und Zöliakiepatienten häufiger betroffen als solche mit RDS.

Weniger klar als die Zahlenverhältnisse sind die Ursachen für die erhöhte Osteoporosegefahr unter RDS. Eine der diskutierten Hypothesen stellt auf eine erhöhte Serotoninaktivität ab, die den Knochenaufbau hemmt. Möglich ist auch, dass diätetische Restriktionen wie ein Laktoseverzicht - oft in Eigenregie der Patienten vorgenommen - die Kalziumaufnahme einschränken. Drittens könnten zirkulierende Zytokine wie TNF alpha eine Rolle spielen. Jedenfalls ist bereits gezeigt worden, dass die CEDBehandlung mit Infliximab, einem TNF-alpha-Hemmer, die Knochendichte erhöht.

Fazit: Patienten, die an einem Reizdarmsyndrom leiden, laufen erhöhte Gefahr, an Osteoporose zu erkranken. Dadurch bedingte Frakturen treten bei ihnen häufiger auf. „Es ist daher zu empfehlen“, resümieren die Autoren der vorliegenden Studie, „die Betroffenen einem entsprechenden Screening zu unterziehen."

Dr. Robert Bublak

Stobaugh DJ et al. Increased risk of osteoporosis-related fractures in patients with irritable bowel syndrome. Osteoporos Int 2012; doi: 10.1007/s00198-012-2141-4 